\title{
Analysis of Dynamic Characteristics -Using Cast Iron and Mineral Resin as Computer Numerical Control Grinder Base Material
}

\author{
Jui-Chang Lin $^{1}$ and Cheng-Jen Lin ${ }^{2 *}$ \\ ${ }^{1}$ Department of Mechanical Design Engineering, National Formosa University Yunlin 632, Taiwan \\ ${ }^{2}$ Institute of Mechanical and Electro-Mechanical Engineering, Department of Power Mechanical Engineering, \\ National Formosa University, Yunlin 632, Taiwan
}

(Received November 11, 2017; accepted January 9, 2018)

Keywords: CNC grinder machine, mineral resin base, cast iron base, modal analysis

Of all the components composing a machine tool, the base is one of the most important parts. It not only props up the weight of all the other components but also withstands the cutting force of processing. The subjects of this study are two grinders; although the aim of their use is slightly different, the structures of the two grinders are very similar. The main variation is the base; cast iron material is used in one base while the other is cast from mineral resin. This study focuses on the similarity of the structures and the differences in dynamic response characteristics caused by different materials. Experimental results showed that possible structural blemishes exist in the mineral resin base in the lower frequency range, and the dynamic characteristics of a mineral resin grinder are worse than those of a cast iron grinder. However, in the frequency range under discussion, the mineral resin grinder is less likely to cause resonance.

\section{Introduction}

To satisfy customers' needs, machine tool vendors aim to develop new generation models with precision and high speed as well as systematization in the highly competitive market. As a result, precisely improving the size and roughness of the surface of a workpiece has become a main concern for grinding. Grinding equipment is necessary in the processing industry because it can be applied to different industries such as semiconductors, aerospace, automotive, and electrical. In the past, a grinder was only used to grind the surface or interior circle; however, grinders now have been developed as computer numerical control (CNC) universal cylindrical grinders.

In $\mathrm{CNC}$ grinder processing, there are factors that (e.g., temperature of the working environment, error compensation of structures, servo systems, electronic control systems, wheel systems, and cooling systems) determine the degree of precision of a workpiece, and the demand for machining accuracy of a grinder is more important than for that of other machine

*Corresponding author: e-mail: 1cj@nfu.edu.tw

http://dx.doi.org/10.18494/SAM.2018.1823 
tools. Hence, if the various factors that affect grinders could be addressed, especially the problems of structural rigidity and vibration, then a grinder's precision would be improved directly. Regarding the problem of grinder structure, the practice in the past has been based on experience with the designers or a group. Now engineers use computer aided engineering software to understand the problems of structure and vibration; therefore, engineers can propose solutions for these problems, while at the same time eliminating the trial and error method, and thereby significantly reducing the design time and costs.

The stability of a grinding machine is an important feature to consider when designing the structure. As the grinder operates, stress concentration in some areas may result from the improper design of the structure. In addition, a machine's structural strength may be insufficient to handle the stress, and the frequency on the machine may magnify vibrations. This abnormal vibration may lead to adverse effects like faulty products, deformation, and fatigue of structure, which shorten the lifespan of the machine. Consequently, to enhance a grinder's precision, several factors should be carefully considered. In this study, the authors focus on the modal parameters of grinders and the base material casting by cast-iron and mineral resin.

There has been a long history of using of cast-iron, and people know much about the characteristics of this material. Undoubtedly, cast-iron is a good choice to be the material of a machine base under condition where the demands are not very high; however, with the demand for precision increasing, it is clear that cast-iron is unable to meet modern needs. Therefore, seeking other alternative materials has become the latest development trend.

Using a highly rigid mineral as a base material is a new option; however, natural minerals are difficult to process, and special requirements for the geometric shapes are not easy to meet. The compromise is to use a granular mineral combined with adhesive and to cast it into a specific geometry to complete the manufacture of the base. At the 2015 EMO toolmaking industry exhibition in Milan, about $25 \%$ of the manufactured units were made of minerals, and this situation has been increasing in recent years.

In terms of research on using minerals, Cho studied the application of mineral resin material in machine beds and used a mixture of steel and mineral resin composites. The results showed that using such materials could significantly improve the damping characteristics and rigidity of machines and could markedly reduce the weight of the machines. ${ }^{(1)}$ Based on the characteristics of polymerized concrete, Suh and Lee designed and manufactured a high-speed CNC milling machine bed to verify its feasibility. ${ }^{(2)}$ Elbestawi analyzed processing methods by combining high-speed milling and grinding, and obtained the modes and natural frequencies of the components via finite element analysis. The dynamic characteristics of the damping and joining surfaces were obtained by dynamic testing of the sample machine, were revised, and then used to verify the machine dynamics model. ${ }^{(3)}$ In the resin-based mineral composites, Song and Hwang tried to use toughening reinforcement materials such as glass fiber, carbon fiber, and steel fiber to enhance mechanical properties. ${ }^{(4)}$ Cameiro et al. studied how to improve the bonding between fiber (glass, carbon, and steel) and substrate material, and then to ensure the resin-based mineral composite material has certain strength and rigidity. ${ }^{(5)}$

Because previous studies focused on a single machine, this work examines two grinders of similar composition, the bases of which are made of either cast iron or mineral resin. In 
the experiment of this study, a three-axis accelerometer is used to measure the vibration, or acceleration of motion of a structure. The force caused by vibration or a change in motion (acceleration) caused the piezoelectric material in accelerometer to be squeezed, which produces an electrical charge that is proportional to the force exerted upon sensor. Since the charge is proportional to the force, and the mass is a constant, then the charge is also proportional to the acceleration. By the characteristics of accelerometer, in experiments, accelerometer could extract vibration signal in different materials, and with this information to do further modal analysis.

\section{Background}

As the mineral machines are made by different manufacturers, their composition and processes are not the same. In this study, the mineral resin base of manufacturer used technology from the German company RAMPF. The main components of mechanical engineering mineral castings are resin binder, filling material, and additives mixed in accordance with the required ratio. The main ingredients of the filling material are basalt stone, granite, quartz, and a variety of sand, and the castings are made by dosing, mixing, casting, compacting, hardening, and forming.

The results of the comprehensive study show that the mineral resin material is better than the cast iron in the following ways:

1. The density of mineral resin is about $33 \%$ of that of cast iron, which means it is relatively lighter, and lowers the compressive strength and modulus of elasticity. Usually, these factors can be improved by increasing the wall thickness of the structure.

2. Thermal conductivity is only about $4 \%$ of that of cast iron; temperature changes have significantly less influence on the dimensional accuracy of the machine tool.

3. Line contraction is only about $2.5 \%$ of that of cast iron, which means that the final molding accuracy is higher than cast iron.

4. Mineral casting has good corrosion resistance to oil, coolant, and other corrosive liquids.

5. In the design, all kinds of oil or electrical lines, containers, and threads can be embedded in the structure, which is difficult to achieve with cast iron.

For this study, we removed sheet metal and some nonessential components; the computer aided design (CAD) drawings of the two grinders are shown in Fig. 1. In this work, the overall test was carried out in experiments as well as simulations. In the simulation, CAD software (SOLIDWORKS) was used to simplify the model of the grinders. After the model was imported into the computer-aided engineering (CAE) software (ABAQUS), the mechanical properties of the materials were given and the binding conditions set between parts. After that, in the finite element method (FEM), the correlation calculation was carried out to obtain each modal frequency and vibration mode. The mechanical properties of the main materials used in these machines are listed in Table 1, and the model revealed in the CAE software is shown in Fig. 2.

In the experiments, this study placed a three-axis accelerometer on the machine to measure the percussion signal at multiple points. Using the spectral analyzer to analyze relevant information, the experimental data was recorded by the computer. This information was 


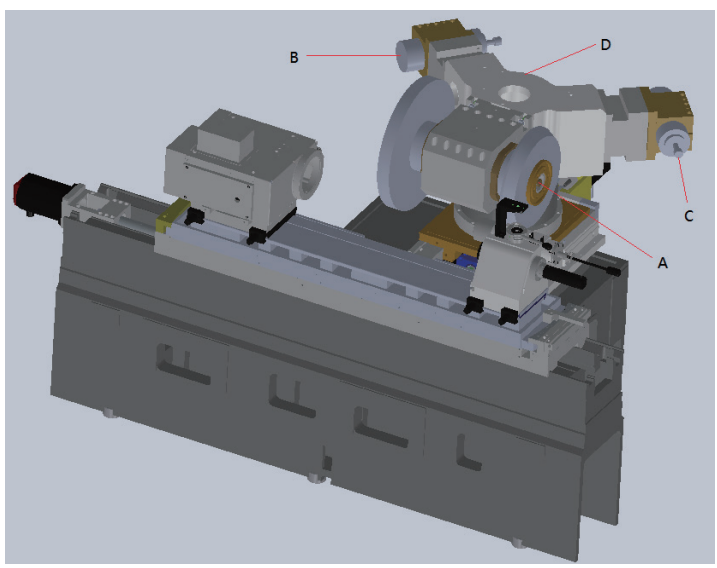

(a)

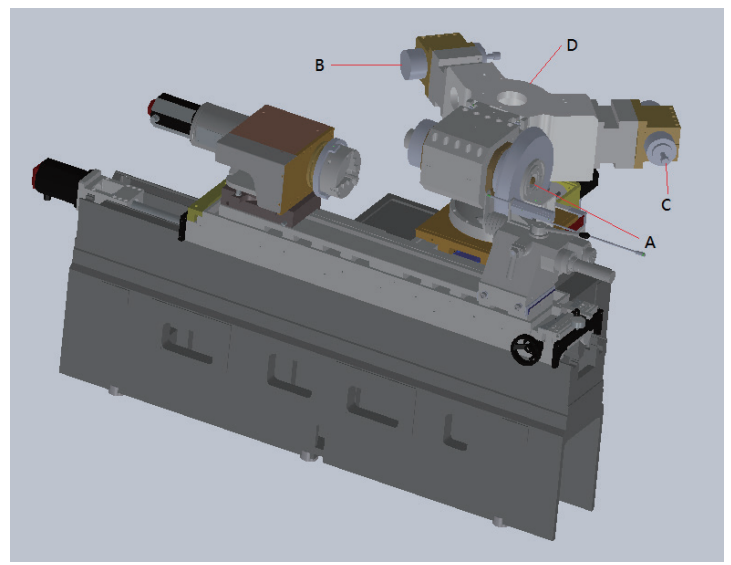

(b)

Fig. 1. (Color online) (a) Grinder with cast iron base. (b) Grinder with mineral resin base. The grinding wheel motors are marked as $\mathrm{A}, \mathrm{B}$, and $\mathrm{C}$; the speeds are $\mathrm{A}=6000 \mathrm{rpm}, \mathrm{B}=24000 \mathrm{rpm}$, and $\mathrm{C}=36000 \mathrm{rpm}$. The mark D is the rotary table of each grinder.

Table 1

Mechanical properties of materials used in the two grinders.

\begin{tabular}{lccc}
\hline Material & Medium carbon-steel & Cast iron & Mineral resin \\
\hline Density (ton $/ \mathrm{mm}^{3}$ ) & $7.89 \mathrm{E}-9$ & $7.2 \mathrm{E}-9$ & $2.4 \mathrm{E}-9$ \\
Young's modulus (Gpa) & 200 & 110 & 40 \\
Poisson's ratio & 0.3 & 0.28 & 0.28 \\
\hline
\end{tabular}

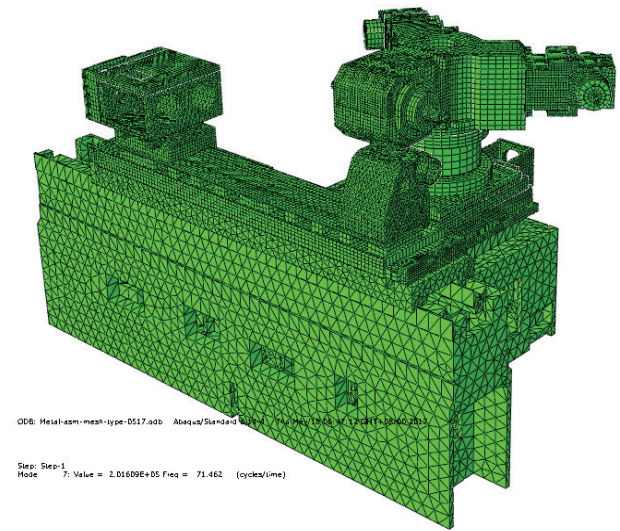

(a)

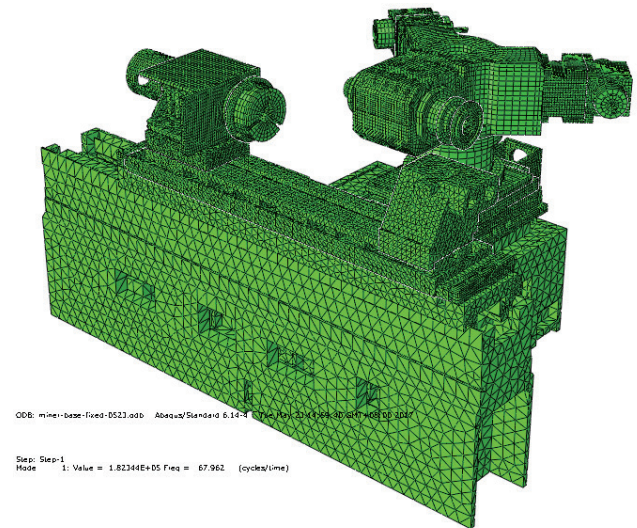

(b)

Fig. 2. (Color online) (a) Trellis diagram of the grinder machine with the cast iron base. (b) Trellis diagram of the grinder machine with the mineral resin base.

imported into ME'scope to get the final result. In order to enhance the effect of comparison of experiment and simulation, the CAD drawing imported into ME'scope was the same as the drawing used in FEM simulation. The equipment for the experiment is listed in Table 2. The difference in appearance of the surfaces of the two materials is shown in Fig. 3. Finally, 
Table 2

Experimental equipment and specifications.

\begin{tabular}{lc}
\hline Experimental equipment & Type \\
\hline Knocking hammer & B\&K, model: 8208 (51771) \\
Three-axis accelerometer & B\&K, model: 4506B \\
Spectral analyzer & B\&K, model: 3560C \\
\hline
\end{tabular}

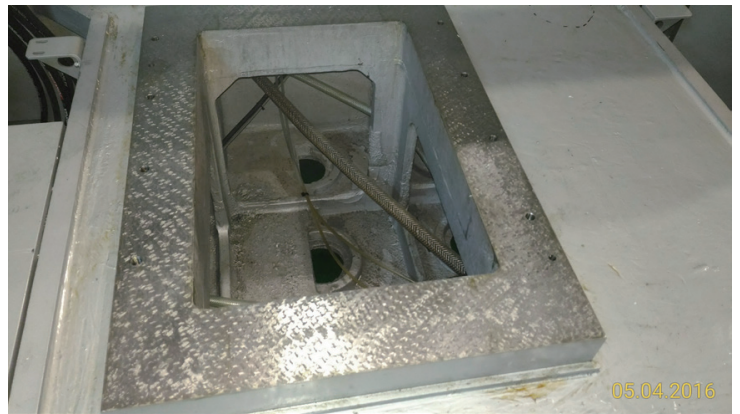

(a)

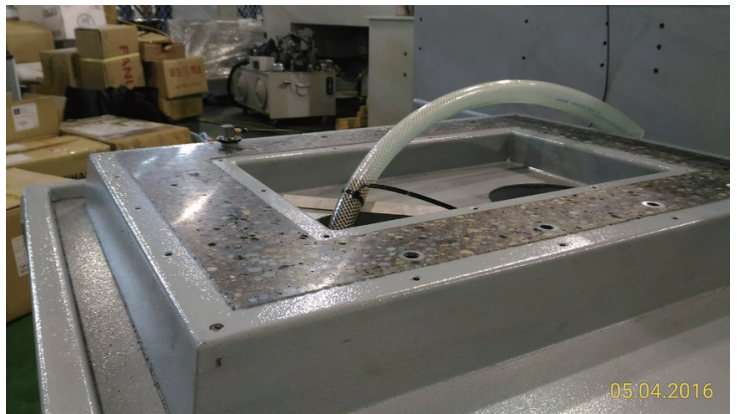

(b)

Fig. 3. (Color online) (a) Base of the grinder is cast with cast iron. (b) Base of the grinder is cast with mineral resin.

the resonance frequency and mode shape obtained by simulation and experiment would be individually revealed.

\section{Results and Discussion}

The following is a comparison between experiments and simulations using the two machines. In general, the machine tool modal analysis frequency value was not set too high. However, as shown in Fig. 1, the highest speed of the grinding machine was $36000 \mathrm{rpm}$; therefore, the modal frequency could be set to $600 \mathrm{~Hz}$ during the simulation. The results of the experimental analysis follow accordingly.

\subsection{Experimental results}

The results of the experiment are shown in Table 3. The minimum resonant frequency of the machine made of mineral resin was almost the same as that of the machine made of cast iron. Generally speaking, the base of machine made by cast iron should be slightly higher. In studying the results of the percussion experiment, we discovered unusual sound in some areas of the mineral resin base by chance; caverns seem to exist in the base structure. Calling on life experience to make a judgment about this observation, we suggest that the item may not be in a homogeneous state when this occurs.

Because the upper structures of the two grinders are almost the same, the presence of unknown holes in the base may be the cause of such a result. But this is only speculation, as we cannot cut the base structure apart to explore the reasons for this result. Although 
Table 3

The experimental results from the two grinders. ${ }^{(6)}$

\begin{tabular}{cccccccc}
\hline \multicolumn{5}{c}{ Cast iron base } & & \multicolumn{2}{c}{ Mineral resin base } \\
\cline { 1 - 3 } \cline { 1 - 2 } Mode & Frequency $(\mathrm{Hz})$ & Mode & Frequency $(\mathrm{Hz})$ & & Mode & Frequency $(\mathrm{Hz})$ \\
\hline 1 & 31.7 & 12 & 256 & & 1 & 31 \\
2 & 48.7 & 13 & 286 & & 2 & 54.6 \\
3 & 58.6 & 14 & 320 & & 3 & 84.8 \\
4 & 85 & 15 & 421 & & 4 & 125 \\
5 & 94 & 16 & 467 & & 5 & 131 \\
6 & 108 & 17 & 512 & & 6 & 160 \\
7 & 118 & 18 & 525 & & 7 & 208 \\
8 & 145 & 19 & 595 & & 8 & 314 \\
9 & 172 & & & & 9 & 331 \\
10 & 188 & & & & 10 & 374 \\
11 & 224 & & & & & \\
\hline
\end{tabular}

the experimental results are not as expected, they conveyed other information. In the lower frequency range under discussion, comparing the modal numbers in which the structures resonated, that of the mineral resin machine was significantly lower than that of the machine made of cast iron. This means that within the lower frequency range, the mineral resin has less chance of developing resonance. Generally, low frequency resonance excitation source usually comes from the grinder's own feed mechanism or grinding wheel system.

The observation of fewer resonant frequencies has been extended to the frequency range under discussion; this means that the mineral resin machine has good shock-absorbing capabilities for most grinding operations. If one considers the same external excitation source acting on the different machines, for the display of the modal shape, only the first and third modes of the cast iron machine are compared with the first and second modes of the mineral resin machine. The results are shown in Fig. 4. From the modal shape shown, at similar resonant frequencies, the strength of the mineral resin machine is indeed relatively lower, but because their structures are similar, the locus of maximum amplitude is quite consistent.

\subsection{Results of simulations}

The simulation of the dynamic characteristics of the two machines is shown in Table 4. Because the results were produced by numerical calculation, a higher number of modes is available, and the frequency values of the lowest mode are significantly higher than those in the experimental results. This may be due to the simplification of the behavior before the simulation. After all, all the components are installed before experiment; it is not easy for the actual situation of the simulation analysis to be shown. As for the modal shape by simulation, the first two modal shapes are a comparison of the two machines. The results are shown in Fig. 5.

From the modal shape shown in Fig. 5, for the same modal number, there are obvious differences between the modal shapes of the two grinders, and it is believed that such variations were caused by using the mineral resin material. But the simulation is just an ideal case, and 


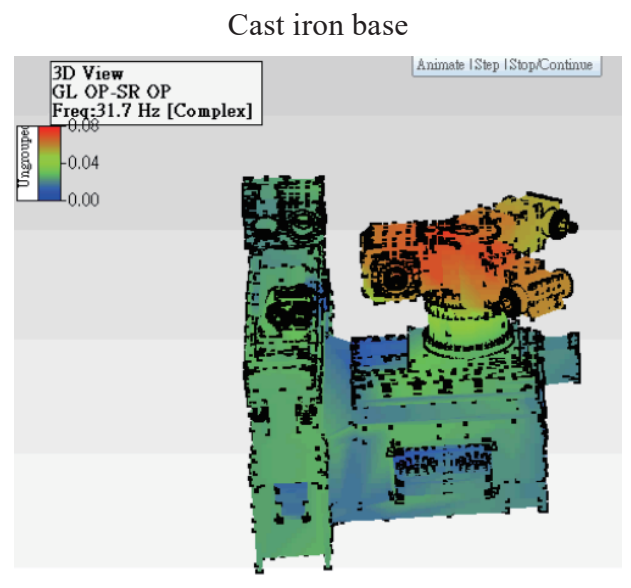

(a)

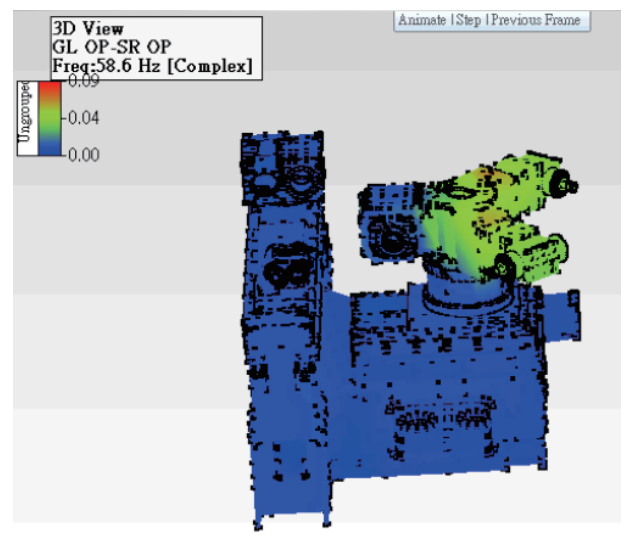

(c)

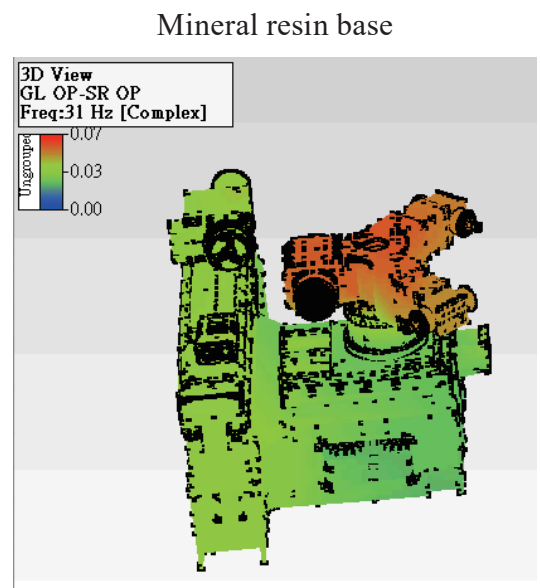

(b)

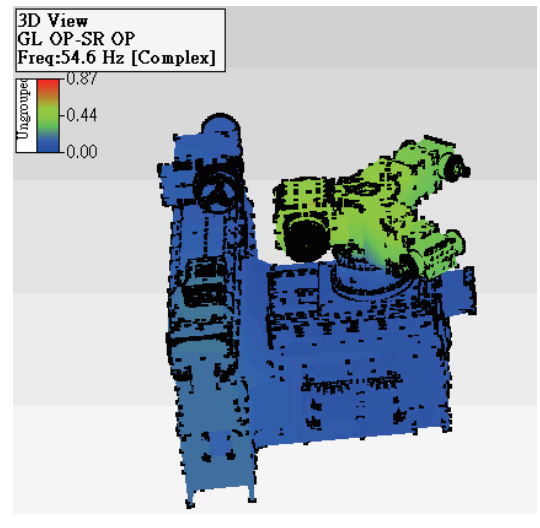

(d)

Fig. 4. (Color online) Comparison of modal shape by experiment: (a) cast iron base at $31.7 \mathrm{~Hz}$, (b) mineral resin base at $31 \mathrm{~Hz}$, (c) cast iron base at $58.6 \mathrm{~Hz}$, and (d) mineral resin base at $54.6 \mathrm{~Hz}{ }^{(6)}$

Table 4

Simulated results from two grinders (the unit of frequency is $\mathrm{Hz}$ ). ${ }^{(6)}$

\begin{tabular}{ccccccccc}
\hline & \multicolumn{3}{c}{ Cast iron base } & & \multicolumn{5}{c}{ Mineral resin base } \\
\cline { 1 - 3 } \cline { 7 - 9 } Mode & Frequency & Mode & Frequency & & Mode & Frequency & Mode & Frequency \\
\hline 1 & 72.4 & 17 & 416.5 & & 1 & 44.9 & 17 & 425.4 \\
2 & 81.9 & 18 & 443.2 & & 2 & 52.1 & 18 & 458.2 \\
3 & 94.4 & 19 & 462 & & 3 & 60.7 & 19 & 467.9 \\
4 & 136.4 & 20 & 485.6 & & 4 & 99.5 & 20 & 491.9 \\
5 & 154 & 21 & 495.4 & & 5 & 106.9 & 21 & 507.4 \\
6 & 159.6 & 22 & 508.3 & & 6 & 116.8 & 22 & 509.3 \\
7 & 172.8 & 23 & 525.7 & & 7 & 131.7 & 23 & 519.3 \\
8 & 206.3 & 24 & 534 & & 8 & 164.2 & 24 & 526.6 \\
9 & 232.3 & 25 & 542.2 & & 9 & 192.7 & 25 & 538.7 \\
10 & 261.1 & 26 & 555.1 & & 10 & 198.9 & 26 & 552.2 \\
11 & 287.6 & 27 & 560.1 & & 11 & 253.9 & 27 & 557.5 \\
12 & 320 & 28 & 570.9 & & 12 & 292.8 & 28 & 567.6 \\
13 & 345.5 & 29 & 578.9 & & 13 & 304.5 & 29 & 575.6 \\
14 & 369.9 & 30 & 590.8 & & 14 & 363.6 & 30 & 584.5 \\
15 & 398.7 & 31 & 596.9 & & 15 & 366 & 31 & 586 \\
16 & 401.7 & - & - & & 16 & 392.5 & 32 & 597.6 \\
\hline
\end{tabular}




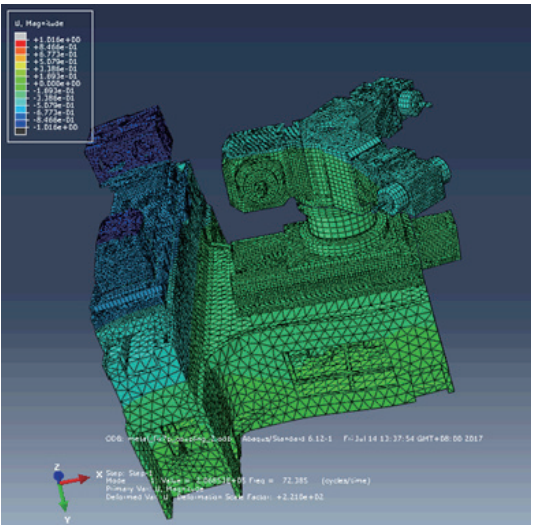

(a)

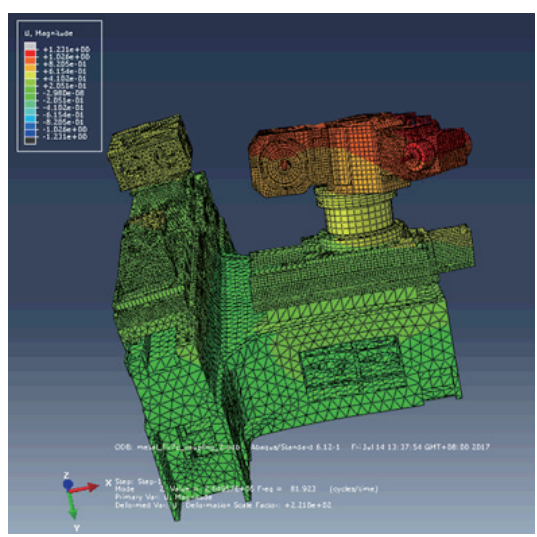

(c)

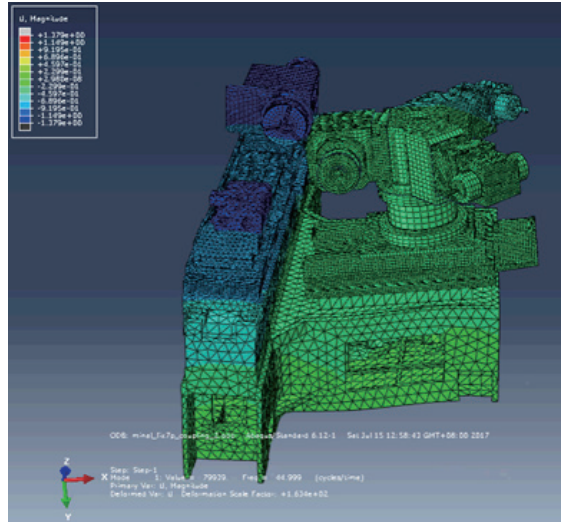

(b)

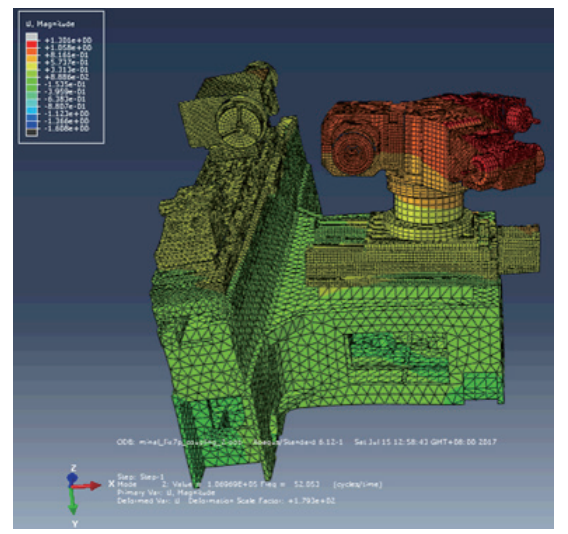

(d)

Fig. 5. (Color online) The comparison of modal shape by simulation: (a) cast iron base at $72.4 \mathrm{~Hz}$, (b) mineral resin base at $44.9 \mathrm{~Hz}$, (c) cast iron base at $81.9 \mathrm{~Hz}$, and (d) mineral resin base at $52.1 \mathrm{~Hz}{ }^{(6)}$

from the previous experiment, there was reason to support the idea that the mineral resin base was not a homogenous construction. Its results do not exactly match the results from the homogenous hypothesis in the simulation. If the overall homogeneity of the mineral resin base can be improved, the results in Fig. 5 also show that the two grinders have greater vibrational amplitude on the rotary table. This is consistent with the experimental results.

\section{Conclusions}

The purpose of this research is to realize the differences between the grinder bases made of cast iron and mineral resin. To summarize the results of the experiments and simulations, the authors' views on the results of modal analysis are as follows.

1. Experimentally, the mineral resin base did not demonstrate better vibration suppression capacity than the cast iron base in the lower frequency range. Aside from the suggestion of inhomogeneity, the composition of the mineral resin and the process are possible causes. On 
the other hand, according to the sound of percussion, the possibility that the material does not fit closely with the steel plate as a mold cannot be excluded.

2. For all possible performing ranges of grinders, the modal number is deficient in the experimental data; thus using, mineral resin can still diminish the possibility of resonance.

3. As shown in Fig. 4, though the modal shapes are similar, an area with larger amplitude arises; the grinder made from mineral resin is remarkably higher than that of cast iron. In brief, the stiffness of mineral resin base is less than that of a cast iron one, which could be predicted based on the elastic modulus of the material.

4. Whether the simulated or experimental results are invoked, in the lower frequency range, the two grinders have a large modal shape on the rotary table. Future improvement should be focused on strengthening the rigidity of the rotary table to ensure the stability of the processing quality.

5. In terms of structural design, the ease of embedding pipes and containers in a structure could be an advantage of mineral resin, but if the embedded subjects are not arranged correctly, the fluidity of the mineral resin during the casting process could be influenced, and the production of interior caves in the structure may become unavoidable.

\section{References}

1 S. K. Cho: Compos. Struct. 93 (2011) 492.

2 J. D. Suh and D. G. Lee: Springer Sci. Bus. Media 4 (2008) 113.

3 M. A. Elbestawi: Ann. CIRP 51 (2002) 307.

4 P. S. Song and S. Hwang: Constr. Build. Mater. 18 (2004) 669.

5 J. A. Cameiro, P. R. L. Lima, and M. B. Leite: Cement Compos. 46 (2014) 65.

6 C. J. Lin: Ph.D. dissertation, National Formosa University (2017). 